\title{
The use of all-oral direct-acting antivirals in hepatitis $C$ virus-infected patients with substance use disorders
}

\author{
Xinyi Jiang, PhD; Hyun Jin Song, PhD; Wei Wang, PhD; Linda Henry, PhD; Lindsey M Childs-Kean, PharmD; \\ Vincent Lo Re III, MD; and Haesuk Park, PhD
}

\section{What is already known about this subject}

- Clinical trials reported that hepatitis $\mathrm{C}$ virus (HCV)-infected patients with substance use disorders (SUDs) who were treated with all-oral direct acting antivirals (DAA) achieved equally high sustained virologic response rates compared to HCVinfected patients without SUDs.

- The American Association for the Study of Liver Diseases and the Infectious Diseases Society of America recommends timely DAA treatment for all patients with HCV, including patients with SUDs.

- High drug costs have led insurers to restrict access to DAA treatments, requiring patients to meet specific prior authorization approval (e.g., abstinence from drug or alcohol use).

\section{What this study adds}

- Only one-third of newly diagnosed patients with chronic HCV filled their prescription for DAA therapy following their HCV diagnosis regardless of SUD status.

- After adjusting for demographics and clinical characteristics, HCV-infected patients with SUDs were significantly less likely to initiate DAA treatments compared to those without SUDs (24\% vs. $34 \%, P<0.01)$.

- Once patients initiated DAA therapy, the overall discontinuation rate was low (3\%) and there was no difference in discontinuation of DAA treatment between those with and without SUDs ( $4 \%$ vs. $3 \%$ ).

\section{ABSTRACT}

BACKGROUND: There is evidence that barriers exist for the initiation of direct-acting antiviral (DAA) treatment for hepatitis $C$ virus (HCV) for those with substance use disorders (SUDs). However, real world clinical evidence of DAA treatment initiation following receipt of a prescription and continuation among those with SUDs and HCV is lacking.
OBJECTIVES: To (1) compare HCV treatment initiation (prescription fill) rates and early discontinuation rates between HCV-infected patients with and without SUDs in the DAA era, and (2) identify patient-level factors associated with HCV treatment initiation and early discontinuation in patients with SUDs.

METHODS: A retrospective cohort analysis of the MarketScan databases (January 2012-December 2018) was conducted for

\section{Author affiliations}

Xinyi Jiang, PhD; Hyun Jin Song, PhD; Wei Wang, PhD; Linda Henry, PhD; and Haesuk Park, PhD; University of Florida, College of Pharmacy, Department of Pharmaceutical Outcomes \& Policy, FL. Haesuk Park, PhD; University of Florida, Center for Drug Evaluation and Safety (CoDES), FL. Lindsey M Childs-Kean, PharmD; University of Florida Pharmacotherapy and Translational Research, College of Pharmacy, Gainesville, FL. Vincent Lo Re III, MD; University of Pennsylvania, Division of Infectious Diseases, Department of Medicine and Center for Clinical Epidemiology and Biostatistics, Department of Biostatistics, Epidemiology, and Informatics, Perelman School of Medicine, Philadelphia, PA.

AUTHOR CORRESPONDENCE:

Haesuk Park, 352.273.6261;

hpark@cop.ufl.edu

J Manag Care Spec Pharm 2021;27(7):873-81

Copyright $@ 2021$, Academy of Managed Care Pharmacy. All rights reserved.

newly diagnosed treatment naïve HCVinfected patients (age $\geq 18$ ) with and without SUDs. We used multivariable Cox regression to estimate adjusted hazard ratios (aHRs) with $95 \%$ confidence intervals of treatment initiation and early discontinuation in those with SUDs versus those without.

RESULTS: We identified a total of 29,228 newly diagnosed HCV-infected patients (6,385 with SUDs and 22,843 without SUDs). 
Overall, DAA treatment initiation for patients with SUDs was significantly lower than that for those without SUDs ( $24 \%$ vs $34 \% ; P<0.01)$. After adjusting for demographics and clinical characteristics, patients with SUDs were less likely to initiate DAA treatments than those without SUDs (aHR, 0.87 [0.82-0.92]). There was no difference in discontinuation of DAA treatment between those with and without SUDs (4\% vs 3\%: aHR, 1.13 [0.81-1.60]). Among patients with SUDs $(n=6,385)$, lower rates of initiating DAA treatment was associated with younger age, and comorbidities including alcoholic liver disease (ALD; aHR, 0.44 [0.33-0.57), chronic kidney disease (CKD) (aHR, 0.52 [0.36-0.75]), and hepatitis B virus (HBV; aHR, 0.64 [0.44-0.92]). DAA treatment discontinuation was associated with younger age, ribavirin (RBV) therapy (aHR, 3.78 [2.21-6.47]), and cirrhosis diagnosis (aHR, 2.42 [1.21-4.84]) but not SUD treatment (aHR, 0.68 [0.34-1.34]).

CONCLUSIONS: HCV-infected patients with SUDs had significantly lower treatment initiation rates, especially in young females and those with ALD, CKD, and HBV. No difference was found in DAA discontinuation. However, younger patients with RBV treatment and/or cirrhosis were more likely to stop treatment. Interventions directed towards these groups are needed to enhance DAA initiation and treatment maintenance among HCV-infected patients with SUDs.

Hepatitis $\mathrm{C}$ virus (HCV) infection is the most common chronic blood-borne infection in the United States. The high-risk behavior of injection drug use in this country accounts for a disproportionately large burden of $\mathrm{HCV}$ infection ( $75 \%$ of newly acquired cases and $\sim 50 \%$ of cases of chronic infection). ${ }^{1,2}$ According to data from the Centers for Disease Control and Prevention, the number of cases of acute HCV infection has more than tripled between 2010 and 2016, following significant increases in substance use disorders (SUDs) driven by increased intravenous injection of illicit drugs, abuse of prescription opioids among young persons, and concomitant use of alcohol and illicit drugs. ${ }^{3-6}$

The vast majority of $\mathrm{HCV}$-infected patients with SUDs have not received interferon-based therapy for their $\mathrm{HCV}$ infection due to concerns about influenza-like symptoms and neuropsychiatric adverse events associate with interferon-based therapy. ${ }^{7-9}$ Fortunately, in recent years, $\mathrm{HCV}$ treatment has taken a major step forward with the introduction of highly efficacious, interferon-free, alloral direct-acting antiviral (DAA) treatments, providing therapeutic efficacy in more than $95 \%$ of patients, including patients with SUDs, within 8-12 weeks. $^{10}$ HCV-infected patients with SUDs who were treated with DAAs achieved equally high sustained virologic response rates as treated patients without SUDs. ${ }^{11}$ These new DAA therapies opened up treatment options for HCV-infected patients with SUDs. Nevertheless, high drug costs have led insurers to restrict access to these medications, requiring patients to meet specific prior authorization approvals (eg, abstinence from drug or alcohol use). ${ }^{12,13}$ Thus, patients with SUDs continue to face barriers to treatment. ${ }^{14}$

To explore how large the gap is for the rates of DAA treatment initiation and DAA treatment discontinuation between $\mathrm{HCV}$-infected patients with SUDs and without in the real world, we aimed to compare the DAA treatment initiation and early discontinuation rates between the two groups. Additionally, we examined the factors associated with $\mathrm{HCV}$ treatment initiation and early discontinuation, particularly SUD status.

\section{Methods}

\section{DATA SOURCE}

We conducted an exploratory retrospective cohort study using the Truven Health Analytic MarketScan Commercial and Medicare Supplemental databases which includes complete claims for retirees with Medicare supplemental insurance paid by employers but not those who only receive Medicare as their primary insurance (2012-2018). ${ }^{15}$ Truven is a nationwide administrative claims database containing person-level information for diagnoses, procedures, and prescriptions across all settings including physician outpatient office visits, hospital stays, and pharmacy claims for over 80 million individuals in the commercial dataset and six million individuals in the Medicare Supplement database. Institutional review board approval was obtained from the University of Florida.

\section{STUDY POPULATION}

Identification of Newly Diagnosed Chronic HCV-Infected Patients and Comorbidities. We established the newly diagnosed chronic HCV cohort using October 2013 to December 2018 Truven data as described above while data from October 2012 to September 2013 were used to ensure at least one year of claims prior to HCV diagnosis. We identified patients aged $\geq 18$ years with newly diagnosed chronic $\mathrm{HCV}$ using the International Classification of Diseases, Ninth and Tenth Revision, Clinical Modification (ICD-9-CM) codes and ICD-10-CM codes (Supplementary Table 1, available in online article). A patient was determined to have chronic HCV if they had one inpatient chronic HCV diagnosis or two outpatient diagnoses of chronic HCV on separate days within one year. ${ }^{16}$ Patients were included if they were continuously enrolled in their health plan one year before and 6 months after their first HCV diagnosis. We excluded patients who had decompensated cirrhosis, hepatocellular carcinoma, or liver transplantation or who had undergone 
HCV therapy including interferon-based or DAA-based treatments during the 1-year period prior to the first $\mathrm{HCV}$ diagnosis to make the cohort homogeneous. Patients were followed from the first HCV diagnosis until at the end of their health insurance enrollment or December 31, 2018, whichever came first. In addition, selected comorbidities were also identified by ICD-9-CM/ICD-10-CM codes (Supplementary Table 1).

Identification of HCV-Infected Patients with SUD. The chronic HCV-infected patients were further categorized based on a diagnosis/treatment of SUD (HCV with SUD or HCV without SUD) recorded between one year before and 6 months after the first HCV diagnosis. By adapting a previously validated algorithm, ${ }_{1}^{17}$ patients were considered to have an SUD if they met 1 of 2 criteria: (1) had at least one inpatient or one outpatient claim of drug or alcohol use disorders using ICD-9-CM/ICD-10-CM codes (Supplementary Table 1); or (2) had a record of methadone, buprenorphine, acamprosate, or naltrexone for opioid use disorder or alcohol use disorder. ${ }^{18-20}$

DAA Treatment Initiation Rate. We defined DAAs using the National Drug Codes according to the US Food and Drug Administration (FDA) and American Association for the Study of Liver Diseases (AASLD)/Infectious Diseases Society of America (IDSA) definitions for treatment-naïve patients during the time period of interest (Supplementary Table 2, available in online article). We grouped DAA treatment into two groups: DAA only or DAA with ribavirin (RBV).

Initiation rate for all-oral DAA therapy was defined as the rate of the first DAA prescription without interferon using pharmacy claims, per 1,000 person-months during the study period. For each individual, the person-months of follow-up were calculated from the first HCV diagnosis date to the first DAA prescription, end of enrollment, or December 31, 2018, whichever came first.

DAA Treatment Discontinuation Rate. Of the HCV-infected patients who initiated DAA therapy, patients were excluded from the discontinuation rate analyses if they disenrolled from their health plan during treatment, switched to another DAA therapy, or had zero days' supply of DAA medication. Patients were considered to have discontinued early if the observed treatment duration (summing the number of days from the filling of the first prescription to the end date of the last prescription claim fill date plus days' supply) was shorter than the expected treatment duration (a 30-day gap was allowed as discussed in prior studies). ${ }^{21-23}$ The expected treatment duration was based on the 2017 AASLD/IDSA $\mathrm{HCV}$ treatment guidelines accounting for baseline cirrhosis diagnosis. ${ }^{21}$ For example, we used 8 weeks for sofosbuvir/ ledipasvir among patients without cirrhosis and a 12-week treatment of the other all-oral therapy with and without cirrhosis.

The DAA therapy discontinuation rate (per 1,000 personmonths) was defined as time to the earliest date of a 30-day gap prior to the completion of therapy from the first DAA prescription date.

\section{STATISTICAL ANALYSIS}

Baseline characteristics (one year before the first HCV diagnosis) were compared between HCV-infected patients with and without SUDs using t-tests for continuous variables and chi-square tests for categorical variables.

To compare DAA initiation or discontinuation between HCV-infected patients with and without SUDs, chi-square tests were used. We used multivariable Cox proportional hazards regression models to estimate adjusted hazard ratios (aHRs) with 95\% confidence intervals (CIs) of treatment initiation and early discontinuation in those with SUDs versus those without (the proportional hazard assumption was not violated based on the Shoenfeld residual). Additionally, the other multivariable Cox regressions were separately used to identify factors associated with DAA initiation/early discontinuation in patients with SUDs or those without, controlling for covariates measured one year before the DAA initiation date (the covariates are listed in Supplementary Table 3 , available in online article). Because the 8-week regimen for sofosbuvir/ledipasvir among patients without cirrhosis was not recommended by guidelines until 2016, we performed a sensitivity analysis for discontinuation using 12-week sofosbuvir/ledipasvir during 2013-2015 and 8-week sofosbuvir/ledipasvir during 2016-2018 for patients without cirrhosis. We assessed for multicollinearity using a variance inflation factor (VIF) with a cutoff of either 5 or 10. All analyses were performed using SAS 9.4 (SAS Institute Inc., Cary, NC).

\section{Results}

\section{PATIENT CHARACTERISTICS}

Of 29,228 patients newly diagnosed with HCV, 22\% $(n=6,385)$ had SUDs while $78 \%(n=22,843)$ did not have SUDs (Supplementary Figure 1 and Supplementary Table 1, available in online article). HCV-infected patients with SUDs were more likely to be younger (mean age $43 \pm 17$ vs $56 \pm 11$ years), more likely male (63\% vs $58 \%)$, have cirrhosis $(2.8 \%$ vs $2.4 \%$ ), and were more likely to have depression, schizophrenia/bipolar, epilepsy, and be pregnant compared to $\mathrm{HCV}$-infected patients in the non-SUD group (all $\mathrm{P}$ values 
$<0.001)$. Of HCV-infected patients with SUDs, $56 \%$ had an opioid use disorder, $60 \%$ had other drug-related disorders, and $48 \%$ had an alcohol use disorder. Approximately one-third of individuals with SUDs received some form of medication for SUD.

\section{DAA TREATMENT INITIATION RATE}

Among 29,228 patients with a new diagnosis of $\mathrm{HCV}, 68 \%$ of HCV-infected patients $(\mathrm{n}=19,874)$ did not receive any $\mathrm{HCV}$ treatment. The remaining $32 \%(\mathrm{n}=9,354)$ of $\mathrm{HCV}-$ infected patients received all-oral DAA treatment (Table 1). Overall, of the treated patients, $81 \%$ received DAA only and $19 \%$ received DAA plus RBV. Among the HCV-infected patients with SUDs, 24\% initiated DAA therapy, which was significantly lower compared to the initiation rate of $34 \%$ of HCV-infected patients without SUDs (13 vs 18 per 1,000 person-months; $\mathrm{P}<0.001)$. After adjusting for covariates, chronic HCV-infected patients with SUDs were less likely to initiate all-oral DAAs than those without SUDs (adjusted hazard ratio [aHR], 0.87 [0.82-0.92]; Table 2).

\section{FACTORS ASSOCIATED WITH DAA TREATMENT INITIATION}

Among patients with SUDs $(n=6,385)$, factors associated with a significantly lower probability of initiating DAAs included: being younger [18-35 years old (aHR, 0.85 [0.73$0.99])$ and $36-50$ years old (aHR, 0.80 [0.68-0.95]) vs 51-64 years old]; being female (aHR, 0.81 [0.72-0.91]); having a Health Maintenance Organization (HMO; aHR, 0.80 [0.670.96]) when compared to a Preferred Provider Organization. Having hepatitis B virus (aHR, 0.64 [0.44-0.92]), mental disorders (aHR, 0.69 [0.62-0.77]), cardiovascular disease (aHR, 0.78 [0.66-0.92]), chronic kidney disease (aHR, 0.52 [0.36-0.76]), alcoholic liver disease (aHR, 0.44 [0.33-0.57]), and being pregnant (aHR, 0.58 [0.40-0.86]) were associated with a significantly lower probability of initiating DAAs (Supplementary Table 4, available in online article). In contrast, patients having cirrhosis were more likely to initiate DAA treatment (aHR, 2.68 [2.32-3.10]). However, receiving medication for an SUD was not associated with DAA initiation (aHR, 0.91 [0.80-1.04]). Similar factors were observed among patients without SUDs $(n=22,843$; Supplementary Table 4 , available in online article).

\section{TREATMENT DISCONTINUATION}

Among the 9,354 HCV-infected patients who initiated DAA treatment, $3 \%(n=262)$ of patients discontinued their DAA treatment. Among the SUD group $(n=1,529), 4 \%(n=61)$ discontinued their treatment (of which, $52 \%$ discontinued DAA treatment only and $48 \%$ discontinued DAAs in combination with RBV treatment) providing a crude incidence rate
TABLE 1 Baseline Characteristics for HCV-Infected Patients in Substance Use Disorders (SUD) and Non-SUD Groups $^{\mathrm{a}}$

\begin{tabular}{|c|c|c|c|}
\hline Characteristic & $\begin{array}{l}\text { SUD group } \\
(n=6,385)\end{array}$ & $\begin{array}{c}\text { Non-SUD group } \\
(\mathrm{n}=\mathbf{2 2}, \mathbf{8 4 3})\end{array}$ & $P$ value \\
\hline Age (mean; SD) & $43 \quad(17)$ & $56 \quad(11)$ & $<0.001$ \\
\hline $18-35$ & $2,566(40.2)$ & $1,327 \quad(5.8)$ & \\
\hline $36-50$ & $884(13.8)$ & $3,432(15.0)$ & \\
\hline $51-64$ & $2,590(40.6)$ & $15,034(65.8)$ & \\
\hline$\geq 65$ & $345 \quad(5.4)$ & $3,050(13.4)$ & \\
\hline Gender (male) & $4,029(63.1)$ & $13,146 \quad(57.6)$ & $<0.001$ \\
\hline \multicolumn{3}{|l|}{ Region } & $<0.001$ \\
\hline Northeast & $1,346(21.1)$ & $4,652(20.4)$ & \\
\hline North central & $1,564(24.5)$ & $3,992(17.5)$ & \\
\hline South & $2,581(40.4)$ & $9,850(43.1)$ & \\
\hline West & $838(13.1)$ & $4,100(18.0)$ & \\
\hline Unknown & $56 \quad(0.9)$ & $249 \quad(1.1)$ & \\
\hline \multicolumn{3}{|l|}{ Type of insurance } & 0.002 \\
\hline PPO & $3,471(54.4)$ & $12,492(54.7)$ & \\
\hline HMO & $827(13.0)$ & $3,215(14.1)$ & \\
\hline COMP & $690(10.8)$ & $2,122 \quad(9.3)$ & \\
\hline POS & $457 \quad(7.2)$ & $1,618 \quad(7.1)$ & \\
\hline Others $^{b}$ & $940(14.7)$ & $3,396(14.9)$ & \\
\hline \multicolumn{4}{|l|}{ Comorbidities } \\
\hline CKD & $208 \quad(3.3)$ & $1,183 \quad(5.2)$ & $<0.001$ \\
\hline COPD & $941(14.7)$ & 2,603 (11.4) & $<0.001$ \\
\hline $\mathrm{CVD}^{\mathrm{c}}$ & 762 (11.9) & $3,175(13.9)$ & $<0.001$ \\
\hline Depression & 2,293 (35.9) & 2,365 (10.4) & $<0.001$ \\
\hline Diabetes & $807(12.6)$ & 4,389 (19.2) & $<0.001$ \\
\hline Dyslipidemia & 1,221 (19.1) & $7,070(31.0)$ & $<0.001$ \\
\hline Epilepsy & $171 \quad(2.7)$ & $191 \quad(0.8)$ & $<0.001$ \\
\hline HIV & $118 \quad(1.9)$ & $561 \quad(2.5)$ & $<0.001$ \\
\hline Hypertension & 2,139 (33.5) & $10,075(44.1)$ & $<0.001$ \\
\hline Pregnancy & $209 \quad(3.3)$ & $250 \quad(1.1)$ & $<0.001$ \\
\hline Schizophrenia/bipolar & $733(11.5)$ & $346 \quad(1.5)$ & 0.004 \\
\hline \multicolumn{4}{|l|}{ Other liver disease } \\
\hline Alcoholic liver disease & $146 \quad(2.3)$ & $80(0.35)$ & $<0.001$ \\
\hline $\begin{array}{l}\text { Non-alcoholic } \\
\text { liver disease }\end{array}$ & $204 \quad(3.2)$ & $694 \quad(3.0)$ & 0.52 \\
\hline Hepatitis A virus & $23 \quad(0.4)$ & $49 \quad(0.2)$ & 0.038 \\
\hline Hepatitis B virus & $63 \quad(1.0)$ & $409 \quad(1.8)$ & $<0.001$ \\
\hline
\end{tabular}


TABLE 1 Baseline Characteristics for HCV-Infected Patients in Substance Use Disorders (SUD) and Non-SUD Groups $^{a}$ (continued)

\begin{tabular}{|c|c|c|c|}
\hline Characteristic & $\begin{array}{l}\text { SUD group } \\
(n=6,385)\end{array}$ & $\begin{array}{c}\text { Non-SUD group } \\
(n=22,843)\end{array}$ & $P$ value \\
\hline \multicolumn{4}{|l|}{ Liver severity } \\
\hline Cirrhosis & $181 \quad(2.8)$ & $538 \quad(2.4)$ & 0.029 \\
\hline \multicolumn{4}{|l|}{ Substance use disorder } \\
\hline Opioid & $3,579(56.1)$ & NA & NA \\
\hline Other drug-related & $3,838(60.1)$ & NA & NA \\
\hline Cocaine, heroin & $1,381(21.6)$ & NA & NA \\
\hline $\begin{array}{l}\text { Sedatives, hypnotics, } \\
\text { anxiolytics, tranquilizers, } \\
\text { and barbiturates }\end{array}$ & $936(14.7)$ & NA & NA \\
\hline Stimulants & $665(10.4)$ & NA & NA \\
\hline $\begin{array}{l}\text { Other (un) specified drug } \\
\text { dependence and drug- } \\
\text { induced mental disorders }\end{array}$ & $3,037(47.6)$ & NA & NA \\
\hline Cannabis & $1,289(20.2)$ & NA & NA \\
\hline Hallucinogens & $87 \quad(1.4)$ & NA & NA \\
\hline Alcohol & $3,059 \quad(47.9)$ & NA & NA \\
\hline $\begin{array}{l}\text { Medication for opioid use } \\
\text { disorder or alcohol use } \\
\text { disorder }\end{array}$ & $2,070 \quad(32.4)$ & NA & NA \\
\hline Methadone & $217 \quad(3.4)$ & NA & NA \\
\hline Buprenorphine & $1,654 \quad(25.9)$ & NA & NA \\
\hline $\begin{array}{l}\text { Naltrexone (including } \\
\text { injectable naltrexone) }\end{array}$ & $610 \quad(9.6)$ & NA & NA \\
\hline Acamprosate & $64 \quad(1.0)$ & NA & NA \\
\hline Claims Dataset type & & & $<0.001$ \\
\hline Commercial & $5,942 \quad(93.1)$ & $19,736(86.4)$ & \\
\hline Medicare Supplemental & $443 \quad(6.9)$ & $3,107(13.6)$ & \\
\hline \multicolumn{4}{|l|}{ Follow-up time } \\
\hline Mean (SD), months & $22.4 \quad(14.0)$ & $25.6 \quad(15.7)$ & $<0.001$ \\
\hline
\end{tabular}

${ }^{a}$ Data are reported as numbers (percentages).

${ }^{b}$ Others included basic/major medical, Exclusive Provider Organization (EPO), non-Capitated Point-of-Service (POS) with capitation, CDHP (Consumer-Driven Health Plan), and HDHP (High Deductible Health Plan).

${ }^{\mathrm{C}} \mathrm{CVD}$ includes cerebrovascular disease, coronary artery disease, and peripheral vascular disease.

$C K D=$ chronic kidney disease $; C O M P=C o m p r e h e n s i v e$ insurances:

$\mathrm{COPD}=$ chronic obstructive pulmonary disease; $\mathrm{HCV}=$ hepatitis $\mathrm{C}$ virus; $H I V=$ human immunodeficiency virus; $\mathrm{HMO}=$ Health Maintenance

Organization; $N A=$ not available; $P O S=$ non-Capitated Point-of-Service; $P P O=$ Preferred Provider Organization; $S D=$ standard deviation; $S U D=$ substance use disorders. of 18 per 1,000 person-months. Among the non-SUD group $(n=7,825), 3 \%(n=201)$ of patients discontinued their treatment (of which, $54 \%$ discontinued DAA treatment only and $46 \%$ discontinued DAAs in combination with RBV treatment) providing a crude incidence rate of 12 per 1,000 person-months. After adjusting for covariates, there was no difference in the hazard of discontinuation of DAA treatment for those with or without SUDs (aHR, 1.14 [0.81-1.60]; Table 3). Sensitivity analysis displayed the similar result (Table 3).

\section{FACTORS ASSOCIATED WITH TREATMENT DISCONTINUATION}

Among patients with SUDs who initiated DAA treatment $(n=1,529)$, factors associated with a significantly higher probability of discontinuing DAA treatment included: taking DAAs in combination with RBV (aHR, 3.90 [2.27-6.70]); being 18-35 years old (aHR, 4.90 [2.15-11.16] vs 51-64 years old); having chronic kidney disease (aHR, 4.85 [1.28-18.47]); and having cirrhosis (aHR, 2.59 [1.29-5.18]). However, different types of SUDs or receiving medication for SUD were not associated with early discontinuation of DAA (Supplementary Table 5, available in online article). Among patients without SUD $(n=7,825)$, factors associated with a significantly higher probability of discontinuing DAA treatment included: taking DAAs in combination with RBV (aHR, 3.77 [2.85-5.01]), and having cirrhosis (aHR, 2.53 [1.86-3.43]; Supplementary Table 5). Multicollinearity was not found in any of the models as all VIFs were found to be around 1 .

\section{Discussion}

This retrospective study using real world data has provided important information on DAA treatment initiation (prescription fill) and early discontinuation among chronic HCV-infected persons with and without SUDs. We found that overall, one-third of newly diagnosed patients with chronic HCV filled a prescription for DAA therapy following their HCV diagnosis regardless of SUD status. Second, fewer HCV-infected patients with SUDs filled their prescription for DAA therapy compared to those without SUDs (24\% vs $34 \%)$ especially among HMO recipients. Thirdly, among those who initiated DAA therapy, the overall discontinuation rate was low (2.8\%), and there was no difference in DAA discontinuation noted between those with or without SUDs or by claims datasets or type of insurance after we adjusted for demographics and clinical characteristics.

These significant findings demonstrate that despite the availability of highly effective treatment for chronic $\mathrm{HCV}$ and the receipt of a prescription, treatment initiation remains very low overall and among those with SUDs. 


\section{TABLE 2} Initiation Rates of All-Oral DAA Therapy for HCV-Infected
Patients, Stratified by Presence of SUD

\begin{tabular}{|c|c|c|c|c|}
\hline & $\begin{array}{c}\text { No. of patients } \\
\text { initiated DAA } \\
\text { (\%) }\end{array}$ & $\begin{array}{l}\text { Person- } \\
\text { months }\end{array}$ & $\begin{array}{c}\text { Crude inci- } \\
\text { dence/1,000 } \\
\text { person-months }\end{array}$ & $\begin{array}{c}\text { Adjusted HR } \\
\qquad(95 \% \mathrm{CI})\end{array}$ \\
\hline \multicolumn{5}{|l|}{ Overall } \\
\hline SUD group $(n=6,385)$ & $1,529(24)^{d}$ & 118,202 & 13 & $0.87(0.82-0.92)$ \\
\hline DAA only & $1,242(20)$ & & 11 & \\
\hline $\mathrm{DAA}+$ ribavirin $^{\mathrm{c}}$ & $287 \quad(4)$ & & 2 & \\
\hline Non-SUD group $(n=22,843)$ & $7,825(34)^{d}$ & 438,890 & 18 & Reference \\
\hline DAA only & $6,349 \quad(28)$ & & 15 & \\
\hline DAA + ribavirinc & $1,476 \quad(6)$ & & 3 & \\
\hline
\end{tabular}

\section{Individuals with Medicare supplemental insurance}

\begin{tabular}{l|r|r|r|r}
\hline SUD group $(\mathrm{n}=443)$ & $105(24)$ & 10,110 & 11 & $0.93(0.75-1.14)$ \\
\hline DAA only & $88(20)$ & & 9 & \\
\hline DAA+ribavirinc & $17(4)$ & & 2 & \\
\hline Non-SUD group $(\mathrm{n}=3,107)$ & $863(28)$ & 68,600 & 13 & Reference \\
\hline DAA only & $754(24)$ & & 11 & \\
\hline DAA + ribavirinc & $109(4)$ & & 2 & \\
\hline
\end{tabular}

Individuals with commercial insurance

\begin{tabular}{|c|c|c|c|c|}
\hline SUD group $(n=5,942)$ & $1,424(24)^{d}$ & 108,093 & 13 & $0.86(0.81-0.92)$ \\
\hline DAA only & 1154 (19) & & 11 & \\
\hline DAA + ribavirinc ${ }^{c}$ & $270 \quad(5)$ & & 2 & \\
\hline Non-SUD group $(n=19,736)$ & $6,962(35)^{d}$ & 370,290 & 19 & Reference \\
\hline DAA only ${ }^{b}$ & $5,595 \quad(28)$ & & 15 & \\
\hline DAA + ribavirinc & 1,367 & & 4 & \\
\hline
\end{tabular}

${ }^{a}$ Cox proportional hazard modeling was used to adjust for age, sex, region, health plan, insurance type, diabetes, cardiovascular disease (including cerebrovascular disease, coronary artery disease, and peripheral vascular disease), chronic kidney disease, mental disorders (including schizophrenia/bipolar, depression, and epilepsy), diabetes, pregnancy, alcoholic liver disease, non-alcoholic liver disease, hepatitis A virus, hepatitis B virus, human immunodeficiency virus, and cirrhosis.

${ }^{b} D A A$ only included sofosbuvir, sofosbuvir/ledipasvir, paritaprevir/ritonavir/ombitasvir \pm dasabuvir, elbasvir/ grazoprevir, sofosbuvir/velpatasvir, sofosbuvir/velpatasvir/voxilaprevir, and glecaprevir/pibrentasvir.

${ }^{C} D A A+$ ribavirin (RBV) included sofosbuvir/ledipasvir + RBV, sofosbuvir + simeprevir + RBV, sofosbuvir + RBV, sofosbuvir + daclatasvir+RBV, paritaprevir/ritonavir/ombitasvir \pm dasabuvir+RBV, elbasvir/

grazoprevir + RBV, sofosbuvir/velpatasvir + RBV, sofosbuvir/velpatasvir/voxilaprevir + RBV, and glecaprevir/ pibrentasvir+RBV.

${ }^{d}$ Compared to the non-SUD group, the SUD group was significantly less likely to initiate DAA $(P<0.001$ from chi-square test).

$\mathrm{Cl}=$ confidence interval; $D A A=$ direct-acting antiviral; $\mathrm{HCV}=$ hepatitis $C$ virus; $H R=$ hazard ratio; $R B V=$ ribavirin; $S U D=$ substance use disorders.

The low treatment initiation in the SUD population may be due to factors such as patients' refusal, physicians' concerns, and payer's rejection/rigid eligibility criteria which appears to be most relevant to those who

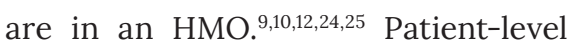
barriers include poor knowledge of $\mathrm{HCV}$, stigma associated with having $\mathrm{HCV}$, mental health issues, and general challenges with accessing the health care system. ${ }^{23,26}$ On the other hand, it is noteworthy that the type of SUDs and the utilization of SUD treatment were not predictors for initiating DAA treatment. In fact, we found that among patients with SUD, factors associated with not initiating treatment included several non-modifiable factors of gender (female) and age (young), which suggests that there is concern about DAA treatment for young female patients with SUDs who are of childbearing age. The determination of when to treat HCV during pregnancy is an area of intense research at the present time, so there are no current recommendations. ${ }^{27,28}$ As such, we recommend that further research is needed to understand what are the barriers within HMO's that are precluding treatment initiation for those who do receive a prescription.

On the other hand, several potential modifiable factors were found to be predictors of not initiating DAA treatment among patients with SUDs. These factors included the type of insurance and certain comorbidities. We found that patients with plans with higher copays (COMP) or with more restrictive coverage (HMO) had lower DAA initiation rates. Such policies may hinder patients' ability to pay for all services, which would suggest that further refinement of cost sharing may be necessary if the goal of hepatitis elimination by the year 2030 is going to be reached. ${ }^{29-31}$ In addition, comorbidities including alcoholic liver disease, chronic kidney disease, and hepatitis B virus were associated with lower rates of DAA initiations among patients with SUDs. More research is now needed to understand barriers to access to DAA treatment among those with the above clinical burdens. ${ }^{15,32}$

A very promising finding was that only less than $3 \%$ of HCV-infected patients who started DAA treatment discontinued treatment regardless of having or not having an SUD, which 


\section{TABLE 3}

Discontinuation Rates of All-Oral DAA Therapy for HCVInfected Patients, Stratified by Presence of SUD $(n=9,354)$

\begin{tabular}{|c|c|c|c|c|}
\hline & $\begin{array}{l}\text { No. of patients } \\
\text { discontinued } \\
\text { DAA (\%) }\end{array}$ & $\begin{array}{l}\text { Person- } \\
\text { months }\end{array}$ & $\begin{array}{c}\text { Crude } \\
\text { incidence } / 1,000 \\
\text { person-months }\end{array}$ & $\begin{array}{l}\text { Adjusted HRa } \\
\qquad(95 \% \mathrm{CI})\end{array}$ \\
\hline \multicolumn{5}{|l|}{ Main analysis } \\
\hline SUD group $(n=1,529)$ & $61(4)^{d}$ & 3,400 & 18 & $1.14(0.81-1.60)$ \\
\hline DAA only ${ }^{b}$ & $32 \quad(2)$ & 2,663 & 12 & \\
\hline DAA+ribavirinc & $29 \quad(2)$ & 738 & 39 & \\
\hline Non-SUD group $(n=7,825)$ & $201(3)^{d}$ & 17,389 & 12 & Reference \\
\hline DAA only ${ }^{b}$ & $108 \quad(2)$ & 13,463 & 8 & \\
\hline DAA + ribavirinc & 93 (1) & 3,926 & 24 & \\
\hline
\end{tabular}

Sensitivity analysis of different treatment duration of sofosbuvir/ledipasvir

\begin{tabular}{l|rl|r|r|l}
\hline SUD group $(\mathrm{n}=1,529)$ & $75(5)^{\mathrm{e}}$ & 3,586 & 21 & $1.08(0.80-1.46)$ \\
\hline DAA only $^{\mathrm{b}}$ & $46(3)$ & 2,848 & 16 & \\
\hline DAA + ribavirinc $^{\mathrm{c}}$ & $29(2)$ & 738 & 39 & \\
\hline Non-SUD group $(\mathrm{n}=7,825)^{\text {DAA only }}$ & $281(4)^{\mathrm{e}}$ & 18,676 & 15 & Reference \\
\hline DAA +ribavirinc $^{\mathrm{c}}$ & $188(2)$ & 14,750 & 13 & \\
\hline
\end{tabular}

Individuals with Medicare supplemental insurance

\begin{tabular}{|c|c|c|c|c|}
\hline SUD group $(n=105)$ & $3 \quad(3)$ & 243 & 12 & $1.90(0.53-6.81)$ \\
\hline DAA only ${ }^{b}$ & 1 (1) & 200 & 5 & \\
\hline DAA + ribavirinc & $2(2)$ & 43 & 47 & \\
\hline Non-SUD group $(n=863)$ & $21 \quad(2)$ & 1,897 & 11 & Reference \\
\hline DAA onlyb & $13 \quad(2)$ & 1,611 & 8 & \\
\hline DAA + ribavirinc & $8 \quad(1)$ & 286 & 28 & \\
\hline
\end{tabular}

\section{Individuals with commercial insurance}

\begin{tabular}{l|rl|r|r|l}
\hline SUD group $(\mathrm{n}=1,424)$ & $58(4)$ & 3,158 & 18 & $1.07(0.75-1.53)$ \\
\hline DAA only $^{\mathrm{b}}$ & $31(3)$ & 2,463 & 13 & \\
\hline DAA + ribavirinc $^{\mathrm{c}}$ & $27(2)$ & 695 & 39 & \\
\hline Non-SUD group $(\mathrm{n}=6,962)^{\text {DAA only }}$ & $180(3)$ & 15,492 & 12 & Reference \\
\hline DAA + ribavirinc $^{\mathrm{c}}$ & $95(2)$ & 11,852 & 8 & \\
\hline
\end{tabular}

${ }^{a}$ Cox proportional hazard modeling was used to adjust for age, gender, region, health plan, insurance type, diabetes, cardiovascular disease (including cerebrovascular disease, coronary artery disease, and peripheral vascular disease), chronic kidney disease, mental disorders (including schizophrenia/bipolar, depression, epilepsy), diabetes, pregnancy, alcoholic liver disease, non-alcoholic liver disease, hepatitis A virus, hepatitis B virus, human immunodeficiency virus, and cirrhosis.

${ }^{b} D A A$ only included sofosbuvir, sofosbuvir/ledipasvir, paritaprevir/ritonavir/ombitasvir \pm dasabuvir, elbasvir/ grazoprevir, sofosbuvir/velpatasvir, sofosbuvir/velpatasvir/voxilaprevir, and glecaprevir/pibrentasvir.

' $D A A+$ ribavirin (RBV) included sofosbuvir/ledipasvir + RBV, sofosbuvir + simeprevir + RBV, sofosbuvir + RBV, sofosbuvir+daclatasvir + RBV, paritaprevir/ritonavir/ombitasvir \pm dasabuvir $+R B V$, elbasvir/

grazoprevir $+R B V$, sofosbuvir/velpatasvir $+R B V$, sofosbuvir/velpatasvir/voxilaprevir $+R B V$, and glecaprevir/ pibrentasvir+RBV.

${ }^{d}$ Compared to the non-SUD group, the SUD group was significantly more likely to discontinue DAA ( $P=0.002$ from chi-square test).

${ }^{e}$ Compared to the non-SUD group, the SUD group was significantly more likely to discontinue DAA $(P=0.014$ from chi-square test).

$\mathrm{Cl}=$ confidence interval; $\mathrm{DAA}=$ direct-acting antiviral; $\mathrm{HCV}=$ hepatitis $\mathrm{C}$ virus; $\mathrm{HR}=$ hazard ratio; $R B V=$ ribavirin; SUD = substance use disorders. was similar to other recent studies which also showed that among those with SUDs those that started treatment were highly likely to complete treatment and to obtain a cure. ${ }^{25,33,34}$ We found that those whose treatment regimens included RBV were almost four times as likely to discontinue therapy regardless of their SUD status as those who did not have RBV as part of their therapy. This finding partially explains why those with cirrhosis, regardless of their SUD status, were almost three times as likely to discontinue treatment. RBV is usually part of the treatment for those with cirrhosis but can cause serious adverse effects (eg, anemia), which may lead patients to stop the treatment earlier. ${ }^{35-37}$

\section{LIMITATIONS}

There are several limitations to this study as this was an analysis of administrative claims data using ICD-9/ ICD-10 codes. However, a recent validation study reported that a coding algorithm including one inpatient or two outpatient ICD-9 codes for $\mathrm{HCV}$ and cirrhosis had high accuracy although using ICD-10 codes has not been validated yet. ${ }^{16}$ It is possible that incomplete, missing, or miscoded claims may impact study findings; however, coding errors are likely to be evenly distributed between the two groups. Another limitation is that we can only identify individuals who engaged the system and received a recorded diagnosis of an SUD or received treatment for an SUD. Thus, we may have underreported the numbers of HCV-infected patients with SUDs. It is also possible that former drug/alcohol use was under-reported which may have biased the HCV without SUDs group, since we were not able to differentiate between patients with former SUDs. Additionally, we cannot generalize our findings to those who are uninsured, Medicaid beneficiaries. 
Despite these limitations, this study provides the first estimates of treatment rates and discontinuation rates for DAA therapy in a nationally representative cohort of commercially insured patients, not geographically limited or representing highly selected patients (eg, opioid treatment clinic).

\section{Conclusions}

Despite the availability of effective all-oral DAA therapies, the overall treatment initiation (prescription fill) rate was only $32 \%$ leaving $68 \%$ of patients with $\mathrm{HCV}$ untreated despite having insurance. This is a concerning finding as patients with delayed access to HCV therapy may experience continued progression of hepatic disease resulting in end-stage liver disease. Besides, HCV-infected patients with SUDs were less likely to fill their DAA prescription, but they were just as likely to complete treatment when compared to those without SUDs. Further research is needed to understand the barriers to treatment for those with SUDs who also have alcoholic liver disease, HBV, or CKD.

\section{DISCLOSURES}

Research reported in this publication was supported in part by the National Institute on Drug Abuse of the National Institutes of Health under award number K01DA045618 (to Park). The other authors have nothing to disclose that may present a potential conflict of interest.

\section{REFERENCES}

1. Hagan $H$. Hepatitis $C$ virus transmission dynamics in injection drug users. Substance use \& misuse. 1998;33(5): 1197-212.

2. Jordan AE, Des Jarlais DC, Arasteh K, McKnight C, Nash D, Perlman DC. Incidence and prevalence of hepatitis $\mathrm{c}$ virus infection among persons who inject drugs in New York City: 2006-2013. Drug and alcohol dependence. 2015;152:194-200.
3. Centers for Disease Control and Prevention (CDC). Surveillence for Viral Hepatitis - United States, 2016. Published 2016. Accessed May 8, 2020. https://www.cdc.gov/hepatitis/ statistics/2016surveillance/commen$\underline{\text { tary.htm }}$

4. Zibbell JE, Asher AK, Patel RC, et al. Increases in Acute Hepatitis C Virus Infection Related to a Growing Opioid Epidemic and Associated Injection Drug Use, United States, 2004 to 2014. American journal of public health. 2018;108(2):175-81.

5. Campbell JV, Hagan H, Latka MH, et al. High prevalence of alcohol use among hepatitis $\mathrm{C}$ virus antibody positive injection drug users in three US cities. Drug and alcohol dependence. 2006;81(3):259-65.

6. Russell M, Pauly MP, Moore CD, et al. The impact of lifetime alcohol use on hepatitis $\mathrm{C}$ treatment outcomes in privately insured members of an integrated health care plan. Hepatology. 2012;56(4):1223-30.

7. Gigi E, Sinakos E, Lalla T, Vrettou E, Orphanou E, Raptopoulou M. Treatment of intravenous drug users with chronic hepatitis $\mathrm{C}$ : treatment response, compliance and side effects. Hippokratia. 2007;11(4):196.

8. Schaefer M, Mauss S. Hepatitis C treatment in patients with drug addiction: clinical management of interferonalpha-associated psychiatric side effects. Current drug abuse reviews. 2008;1(2):177-87.

9. Malespin M, Harris C, Kanar O, et al. Barriers to treatment of chronic hepatitis $\mathrm{C}$ with direct acting antivirals in an urban clinic. Annals of hepatology. 2019;18(2):304-09.

10. Grebely J, Bruneau J, Bruggmann P, et al. Elimination of hepatitis $C$ virus infection among PWID: The beginning of a new era of interferon-free DAA therapy. Int J Drug Policy. Sep 2017;47:26-33.
11. Christensen S, Buggisch P, Mauss S, et al. Direct-acting antiviral treatment of chronic HCV-infected patients on opioid substitution therapy: Still a concern in clinical practice? Addiction. 2018;113(5):868-82.

12. Gowda C, Lott S, Grigorian M, et al. Absolute insurer denial of direct-acting antiviral therapy for hepatitis C: a national specialty pharmacy cohort study. Open Forum Infect Dis. 2018;5(6):ofy076. doi: 10.1093/ofid/ofy076.

13. Re III VL, Gowda C, Urick PN, et al. Disparities in absolute denial of modern hepatitis $\mathrm{C}$ therapy by type of insurance. Clinical Gastroenterology and Hepatology. 2016;14(7):1035-43.

14. U.S. Department of Health \& Human Services. Viral Hepatitis National Strategic Plan. Published 2018. Accessed May 8, 2020. https://www.hhs.gov/hepatitis/viral-hepatitis-action-plan/index. $\underline{\text { html }}$

15. Park H, Wang W, Henry L, Nelson DR. Impact of all-oral direct-acting antivirals on clinical and economic outcomes in chronic hepatitis $\mathrm{C}$ patients in the US. Hepatology. 2019;69(3):1032-45. doi: 10.1002/hep.30303

16. Niu B, Forde KA, Goldberg DS. Coding algorithms for identifying patients with cirrhosis and hepatitis B or $\mathrm{C}$ virus using administrative data. Pharmacoepidemiology and drug safety. 2015;24(1):107-11.

17. Janjua NZ, Islam N, Kuo M, et al. Identifying injection drug use and estimating population size of people who inject drugs using healthcare administrative datasets. International Journal of Drug Policy. 2018;55:31-39.

18. Durand M, Wang Y, Venne F, Lelorier J, Tremblay CL, Abrahamowicz M. Diagnostic accuracy of algorithms to identify hepatitis C status, AIDS status, alcohol consumption and illicit drug use among patients living with HIV in an administrative healthcare database. Pharmacoepidemiology and drug safety. 2015;24(9):943-50. 
19. Missouri Department of Social Services. Substance Use Disorder Diagnoses Codes. Published 2017. Accessed May 8, 2020. https://dss. mo.gov/mhd/cs/pharmacy/pdf/substance-use-disoder-diagnoses-codes.pdf

20. Laine C, Hauck WW, Gourevitch MN, Rothman J, Cohen A, Turner BJ. Regular outpatient medical and drug abuse care and subsequent hospitalization of persons who use illicit drugs. JAMA. 2001;285(18):2355-62.

21. American Association for the Study of Liver Deases (AASLD), Infectious Diseases Society of America (IDSA). HCV Guidance: Recommendations for Testing, Managing, and Treating Hepatitis C: Initial Treatment of Adults with HCV Infection. Updated 2020. Accessed May 8, 2020. https://www.hcvguidelines.org/ treatment-naive

22. Puenpatom A, Hull M, McPheeters J, Schwebke K. Disease burden, early discontinuation, and healthcare costs in hepatitis $\mathrm{C}$ patients with and without chronic kidney disease treated with interferon-free direct-acting antiviral regimens. Clinical drug investigation. 2017;37(7):687-97.

23. Park H, Song HJ, Jiang X, Henry L, Cook RL, Nelson DR. Direct-Acting Antiviral Treatment Use Remains Low Among Florida Medicaid Beneficiaries With Chronic Hepatitis C. Hepatology Communications. 2020.

24. Asher AK, Portillo CJ, Cooper BA, Dawson-Rose C, Vlahov D, Page KA. Clinicians' views of hepatitis $\mathrm{C}$ virus treatment candidacy with direct-acting antiviral regimens for people who inject drugs. Substance use \& misuse. 2016;51(9):1218-23.
25. Jain MK, Thamer M, Therapondos G, et al. Has access to hepatitis $\mathrm{C}$ virus therapy changed for patients with mental health or substance use disorders in the direct-acting-antiviral period? Hepatology. 2019;69(1):51-63.

26. Grebely J, Oser M, Taylor LE, Dore GJ. Breaking down the barriers to hepatitis $\mathrm{C}$ virus (HCV) treatment among individuals with $\mathrm{HCV} / \mathrm{HIV}$ coinfection: action required at the system, provider, and patient levels. J Infect Dis. 2013;207(suppl_1):S19-S25.

27. Cervino L, Hynicka LM. Direct-acting antivirals to prevent vertical transmission of viral hepatitis C: when is the optimal time to treat? Ann Pharmacother. 2018;52(11):1152-57.

28. Freriksen JJ, van Seyen M, Judd A, et al. Review article: direct-acting antivirals for the treatment of HCV during pregnancy and lactation - implications for maternal dosing, foetal exposure, and safety for mother and child. Aliment Pharmacol Ther. 2019;50(7):738-50.

29. Committee on a National Strategy for the Elimination of Hepatitis B and C, Board on Population Health and Public Health Practice, Health and Medicine Division, National Academies of Sciences, Engineering, and Medicine. Eliminating the Public Health Problem of Hepatitis $B$ and $C$ in the United States: Phase One Report. National Academies Press; 2016.

30. National Academies of Sciences, Engineering, and Medicine, Health and Medicine Division, Board on Population Health and Public Health Practice, Committee on a National Strategy for the Elimination of Hepatitis B and C. A National Strategy for the Elimination of Hepatitis B and C: Phase Two Report. National Academies Press; 2017.
31. Xu WY, Shooshtari A, Jung J.

Disparities in cost-related drug nonadherence under the Affordable Care Act. Journal of Pharmaceutical Health Services Research. 2019;10(2):177-85.

32. Butt AA, Yan P, Lo Re III V, Shaikh OS, Ross DB. Trends in treatment uptake and provider specialty for hepatitis $\mathrm{C}$ virus (HCV) infection in the Veterans Affairs Healthcare System: results from the electronically retrieved cohort of $\mathrm{HCV}-$ infected veterans (ERCHIVES). Clin Infect Dis. 2019;68(5):857-59.

33. Terrault NA, Zeuzem S, Di Bisceglie AM, et al. Effectiveness of ledipasvir-sofosbuvir combination in patients with hepatitis $\mathrm{C}$ virus infection and factors associated with sustained virologic response. Gastroenterology. 2016;151(6):1131-40. e1135.

34. Serper M, Evon DM, Stewart PW, et al. Medication Non-adherence in a Prospective, Multi-center Cohort Treated with Hepatitis C Direct-Acting Antivirals. J Gen Intern Med. 2020;35(4):1011-20.

35. Guardigni V, Badia L, Conti M, et al. Liver decompensation predicts ribavirin overexposure in hepatitis $\mathrm{C}$ virus patients treated with direct-acting antivirals. World J Hepatol. 2017;9(34):1270.

36. Miotto N, Mendes LC, Zanaga LP, et al. Predictors of early discontinuation of interferon-free direct antiviral agents in patients with hepatitis $C$ virus and advanced liver fibrosis: results of a reallife cohort. Eur J Gastroenterol Hepatol. 2017;29(10):1149-54.

37. Jung J, Du P, Feldman R, Riley III T. Discontinuation of new hepatitis $\mathrm{C}$ drugs among Medicare patients. Am J Manag Care. 2020;26(2):84-88. 